International Journal of Medical Anesthesiology 2020; 3(1): 184-188

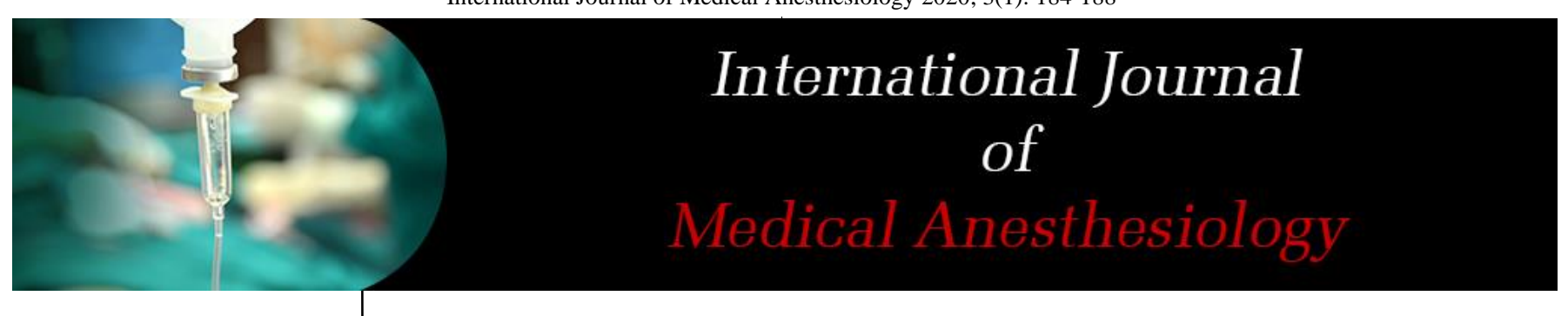

E-ISSN: 2664-3774

P-ISSN: 2664-3766 Www.anesthesiologypaper.com IJMA 2020; 3(1): 184-188 Received: 17-11-2019 Accepted: 21-12-2019

Dr. Sonali M Khobragade Associate Professor, Department of Anaesthesiology, Indira Gandhi Government Medical College and Hospital, Nagpur, Maharashtra, India

Corresponding Author: Dr. Sonali M Khobragade Associate Professor, Department of Anaesthesiology, Indira Gandhi Government Medical College and Hospital, Nagpur, Maharashtra, India

\section{Anaesthetic complications in paediatric patients of cochlear implant surgery: A retrospective study}

\author{
Dr. Sonali M Khobragade
}

DOI: https://doi.org/10.33545/26643766.2020.v3.i1c.88

\section{Abstract}

Background: Cochlear implants play very important role in hearing impairment in adults and children by acting as electromechanical transducer permitting them to integrate with the hearing world. Paediatric Cochlear implant surgery is highly specialized and unique. In this study, we investigated the incidence of anaesthetic complications in paediatric patients for CI.

Methods: A retrospective study was done in 27 paediatric patients who underwent cochlear implant between 2018-2019. Association between two variables were obtained by Fischer exact Chi square test. Observations: Out of 27 paediatric patients, 5 patients had anaesthesia related complications, yielding a complication rate of $18.51 \% .3$ patients had URTI and 2 patients had nausea /or vomiting. There was more risk of respiratory complications in peri-operative period in patients who had history of URTI or LRTI ( $\mathrm{p}=0.049$ ).

Conclusions: General anaesthesia was well tolerated by paediatric patients. Most common complications were respiratory without long term sequelae and managed effectively.

Keywords: Cochlear implant surgery, Paediatric patients, Anaesthesia complications

\section{Introduction}

Cochlear implant, like a true bionic human hair cell, receive mechanical energy and convert it into a series of electrical impulses. The human cochlea being a electromechanical transducer, transforms these electrical impulses to the auditory cortex for generation of the meaningful information so that the cochlear implant recipient of sensori-neural deafness can reintegrate with the hearing world. First cochlear implant was done 30 years back in adult. Cochlear implant is the most sophisticated scientific innovation of medical science which can totally change the life of deaf and dumb children. With proper speech therapy, these children can vocalize and communicate with the surrounding people thereby leading a normal social life. Our, Indira Gandhi Government Medical College is the first medical college in Maharashtra to start and successfully implement cochlear implant surgery workshop under national programme for prevention and control of deafness with the help of ADIP Scheme (Assistance to disabled persons). Cochlear implant is a journey towards meaningful sound. We have successfully completed 27 cochlear surgeries in our centre and proudly the first government medical college in Maharashtra where 27 cochlear implant surgeries have been successfully completed. Out of total 27 children of cochlear implant, 14 were from rural areas especially from tribal areas-12 and 13 from urban areas. All the children with age in the range of 1 year 11 months to 6 years 2 months and all were from economically weaker sections. The cost of CI is approximately 6- 7 Lacs hence this programme has given me and my team a great opportunity to serve the society. Being an anaesthesiologist, there are various anaesthesia concerns. Surgery for cochlear implant is specialized and complicated. So anaesthesia technique plays a very important and crucial role in the successful outcome of these patients.

Challenges in anaesthesia for cochlear implant surgery ${ }^{1}$; a) Paediatric patients. b) Coexisting hereditary and medical diseases such as congenital heart diseases, CNS anamolies, genitourinary diseases, respiratory diseases, Usher syndrome, Pendred syndrome, Waardenberg syndrome, Treacher Collins, Klippel Feil, Jerval and Lange-Neilson CHARGE Syndromecoloboma of eye, cardiomegaly, cho-anal atresia, mental retardation, genital and ear abnormalities etc. c) Anxious parents. d) Special intra and post- operative concerns. e) Anti emesis prophylaxis and f) Analgesia. 
Before cochlear implant there has to be objective assessment of hearing, radiograph of skull and CT/ MRI of temporal bone. Anaesthesiologist plays a important role while doing Computed tomography scan, Magnetic resonance imaging, Brain evoked response audiometry and Cochlear implant of these patients. Surgical technique consists of placement of computerized electric prosthesis via trans-mastoid approach with maintaining the functional integrity of facial and cochlear nerve. Despite the functional gain achieved after cochlear implant by improving the quality of life with cognitive improvement and learning ability, there is always an anxiety in parents regarding surgery and general anaesthesia. Hence this study was done to evaluate the incidence of anaesthesia related complications in children undergoing cochlear implant.

\section{Methodology}

After approval from institutional ethics committee, this retrospective study was done in a tertiary care hospital. Patients who underwent cochlear implant for severe to profound sensori-neural deafness with age less than 10 years during the period of 2018-2019 were included in the study. Preoperative evaluation consisted of thorough evaluation of general condition with medical and surgical illness along with necessary biochemical investigations. Familiarization with the child and his/her parents was the most important part of the assessment. The goal of anaesthesia was smooth induction, maintenance of hemodynamics, adequate regulation of depth of anaesthesia for use of nerve stimulation and evaluation of nerve response time, uneventful extubation and adequate anti-emetic prophylaxis for prevention and treatment of nausea, vomiting and vertigo. Surgery consisted of post aural incision, posterior tympanotomy, cochleostomy with electrode insertion. General anaesthesia was administered to all the patients. Monitoring included was electrocardiogram (ECG), pulse oximetry, non-invasive blood pressure (NIBP), Temperature and End tidal carbon dioxide after intubating the child. All patients received Inj. glycopyrrolate $5 \mathrm{mcg} / \mathrm{kg}$, Inj. Fentanyl $1 \mathrm{mcg} / \mathrm{kg}$, Inj. Midazolam $0.03 \mathrm{mg} / \mathrm{kg}$ prior to induction. The anaesthesia was induced by Inj. Thiopentone $4-5 \mathrm{mg} / \mathrm{kg}$ and Inj. Atracurium $0.5 \mathrm{mg} / \mathrm{kg}$ in 15 children, Inj. Propofol $2 \mathrm{mg} / \mathrm{kg}$ with Inj. Atracurium $0.5 \mathrm{mg} / \mathrm{kg}$ in 5 children and sevoflurane $8 \%$ with Inj. Atracurium $0.5 \mathrm{mg} / \mathrm{kg}$ in 7 children. The anaesthesia was maintained on oxygen and sevoflurane (end tidal MAC- 2) with intermittent doses of Inj. Atracurium $0.2 \mathrm{mg} / \mathrm{kg}$. Inj. Fentanyl was repeated to maintain the depth of anaesthesia and hemodynamics. Maintenance fluid used was Isolyte-P. Analgesia was given with Acetaminophen/ Diclofenac suppository intraoperatively and postoperatively with Syr. Ibugesic plus. Inj. Ondansetron $0.1 \mathrm{mg} / \mathrm{kg}$ and Inj. Dexamethasone $0.25 \mathrm{mg} / \mathrm{kg}$ was given for anti-emetic prophylaxis in all the patients. At the conclusion of surgery, inhalational agent was terminated. The child was reversed with Inj. Neostigmine and Inj. Glycopyrrolate. Tracheal extubation was done after return of reflexes and child fully awake. All patients were kept in high dependency unit for postoperative monitoring. After 4 weeks of surgery, all patients were fitted with external sound processor which was activated by CI audiologist. Data recorded included age of child, weight, side of implantation, medical/surgical history, recent illness, premedication, anaesthetic agents used for induction and maintenance, duration of surgery, medications given, intraoperative and postoperative complications, ASA physical status, intravenous fluids given.

\section{Statistical analysis}

Statistical analysis was done by using Microsoft Excel and SPSS version 21.0. Categorical variables were expressed in percentage. Association between two variables were obtained by Chi Square test and Fischer Chi square exact test. P-value less than 0.05 was considered significant.

\section{Results}

\section{Demographic data}

Total 27 Cochlear implant surgeries were done during the year 2018-2019 at Indira Gandhi Government Medical College and Hospital, Nagpur. The mean age at implantation was 4.17 years with the range of 1 year 11 months to 6 years 2 months. Majority of the patients were in the age group of 4-6 years. Of all these patients, 23 patients (85.19\%) were of ASA class 1 and 4 patients (14.81\%) from ASA class 2 and none from ASA class 3 or 4(Table 2). All patients had unilateral implants. Mean Duration of surgery was 142 minutes (45min- 4hrs)

Table 1: Patient demographics based on Age and American Society of Anaesthesiologists Classification.

\begin{tabular}{|c|c|c|}
\hline Age (in Years) & NO. Of Patients & ASA \\
\hline \multirow{2}{*}{$1-2$} & \multirow{2}{*}{$1(3.7 \%)$} & $\mathrm{A}-1(3.7 \%)$ \\
\cline { 3 - 3 } & & $\mathrm{B}$ \\
\cline { 3 - 3 } $2-4$ & \multirow{2}{*}{$9(33.33 \%)$} & $\mathrm{C}$ \\
\cline { 3 - 3 } & & $\mathrm{A}-8(29.6 \%)$ \\
\cline { 3 - 3 } & \multirow{2}{*}{$16(59.25 \%)$} & $\mathrm{B}-1(3.7 \%)$ \\
\cline { 3 - 3 } & & $\mathrm{C}-0$ \\
\cline { 3 - 3 } & & $\mathrm{A}-13(48.15 \%)$ \\
\hline \multirow{2}{*}{$4-6$} & \multirow{2}{*}{$1(3.7 \%)$} & $\mathrm{C}-3(11.1 \%)$ \\
\hline \multirow{2}{*}{$>6$} & & $\mathrm{~A}-1(3.7 \%)$ \\
\cline { 3 - 3 } & & $\mathrm{B}-0$ \\
\hline Male: Female Ratio & $1.07: 1$ & $\mathrm{C}-0$ \\
\hline \multirow{2}{*}{ Total } & $27(100 \%)$ & $\begin{array}{c}\text { ASA 1-23(85.19\%) } \\
\text { ASA 2-04(14.81\%) }\end{array}$ \\
\hline
\end{tabular}

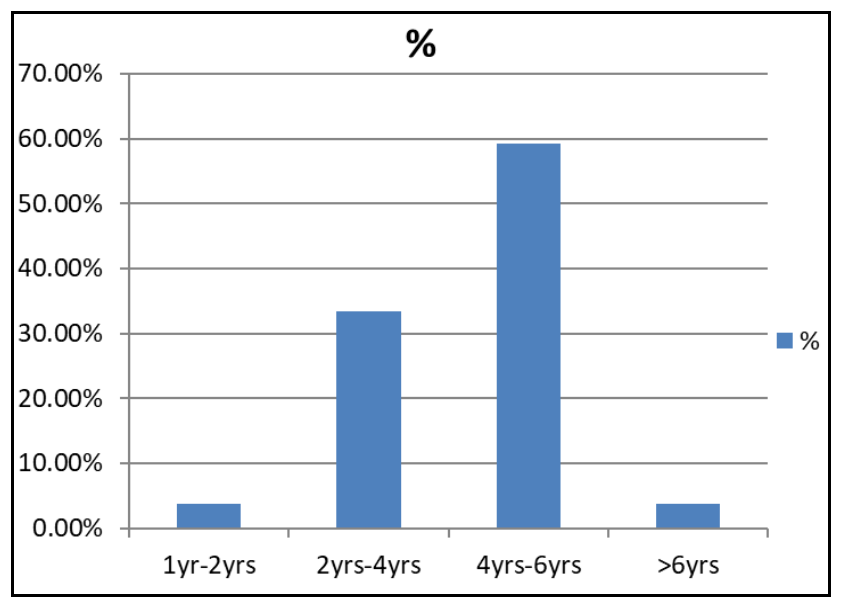

Fig 1: Showing age of patients

\section{Co-morbidities in patients}

There were various co-morbidities associated in children undergoing Cochlear implant. Most common morbidity was history of prematurity $(\mathrm{n}=5,18.52 \%)$ followed by history of neurological abnormality such as meningitis and encephalitis $(n=3,11.11 \%)$, LRTI $(n=3,11.11 \%)$ and URTI $(\mathrm{n}=3,11.11 \%)$. 
Table 2: Depicts the list of co-morbidities.

\begin{tabular}{|c|c|c|}
\hline CO-Morbidities & Number & Percentage \\
\hline Ex-prematurity & 05 & $18.52 \%$ \\
\hline h/o Meningitis/ Encephalitis & 03 & $11.11 \%$ \\
\hline H/O LRTI & 03 & $11.11 \%$ \\
\hline H/O URTI & 03 & $11.11 \%$ \\
\hline Failure to thrive & 01 & $3.70 \%$ \\
\hline Drug allergy (NSAIDS) & 01 & $3.70 \%$ \\
\hline H/O Jaundice & 02 & $7.41 \%$ \\
\hline H/O Congenital Heart Disease (operated for Tetralogy of Fallots with PDA ligation) & 01 & $3.70 \%$ \\
\hline Heart murmur & 02 & $7.41 \%$ \\
\hline H/o NICU at birth & 03 & $11.11 \%$ \\
\hline Asthma & 0 & \\
\hline GERD & 0 & \\
\hline ESRD & 0 & \\
\hline Hip Dysplasia & 0 & \\
\hline HYDROCEPHALUS, Brain tumour & 0 & \\
\hline Abnormal biochemical investigations ANEMIA (Hb $<10 \mathrm{Gm} \%)$ & 02 & $7.41 \%$ \\
\hline
\end{tabular}

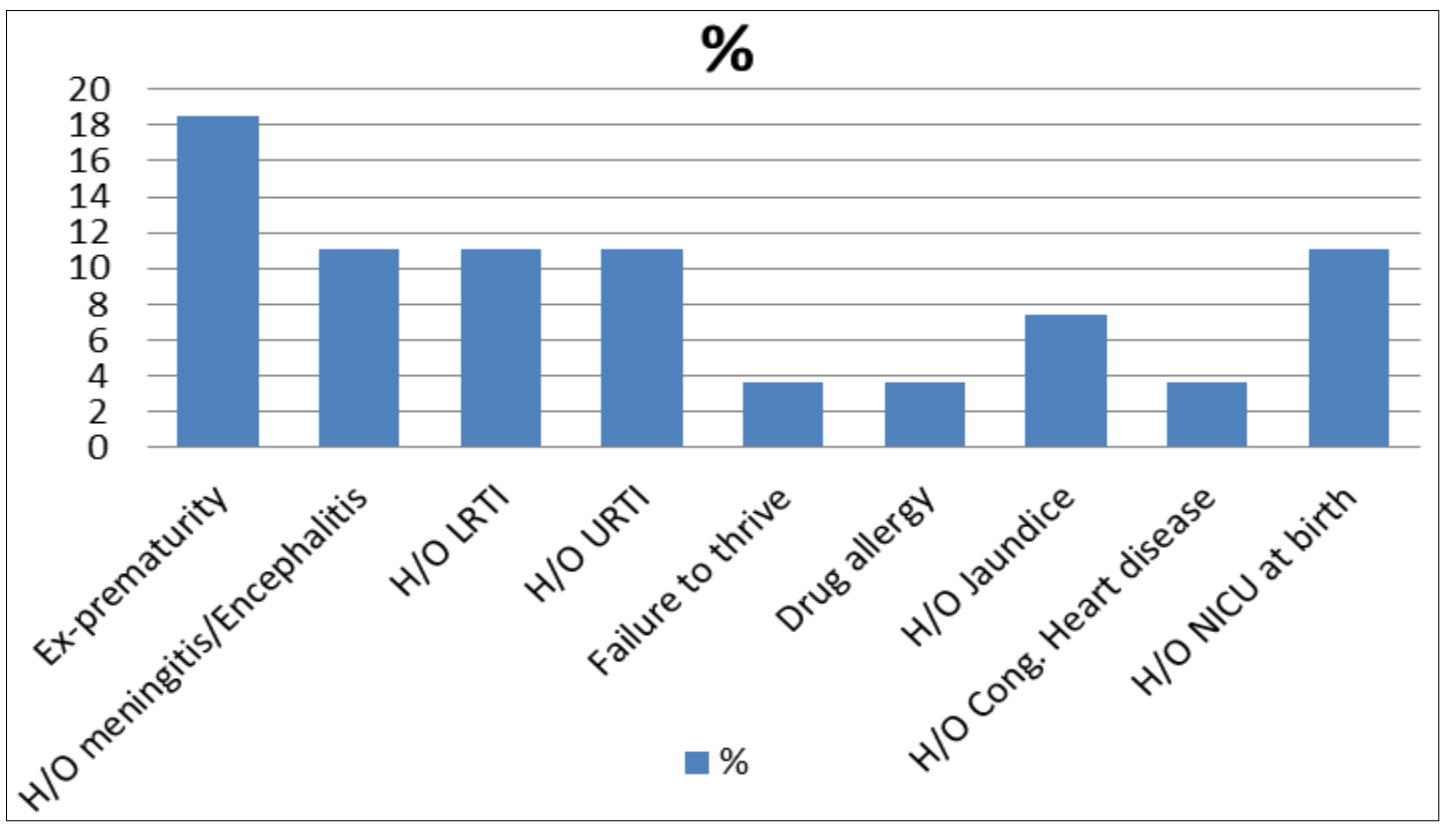

Fig 2: Showing associated co-morbidities

\section{Complications}

No major complications were seen in any child undergoing Cochlear implantation. Total 5 patients had complications out of 27 patients. Most common complications were respiratory complications like cough and upper respiratory tract infection in 3 children $(11.11 \%)$ which was managed with antibiotics, anti-histaminic drugs and nebulization with Duolin effectively. Out of these 3 children, 2 children had H/O URTI and one had H/O LRTI within 4 weeks of surgery. None of the patient had laryngospasm, bronchospasm and stridor during induction or emergence. None of the patient had multiple or traumatic intubation attempts. No patient required prolonged intubation and ventilator support in post-operative period. Analgesia was adequate in all the patients. Nausea and vomiting was seen in 2 children $(7.41 \%)$ which was controlled with Inj. Ondansetron and Inj. Dexamethasone. Fever was seen in 3 children $(11.11 \%)$ which was treated with Paracetamol suppository and antibiotic treatment. 2 patients $(7.41 \%)$ had unilateral lid swelling which was due to tight compressive bandage and subsided slowly after proper loose application of bandage. One child $(3.70 \%)$ had meningismus after surgery which was treated aggressively with higher antibiotics (Inj. Vancomycin and Inj. Piptaz) and meticulous monitoring for 14 days.

Table 3: Depicts the complications in peri-operative period.

\begin{tabular}{|c|c|c|}
\hline Complications & Number of Patients & Percentage \\
\hline Coughing/ URI & 03 & $11.11 \%$ \\
\hline Nausea and vomiting & 02 & $7.41 \%$ \\
\hline Lid swelling & 02 & $7.41 \%$ \\
\hline Meningismus & 01 & $3.70 \%$ \\
\hline Laryngospasm, Bronchospasm, Stridor, CROUP & None & \\
\hline Multiple or Traumatic intubation & None & \\
\hline Prolonged intubation & None & \\
\hline
\end{tabular}




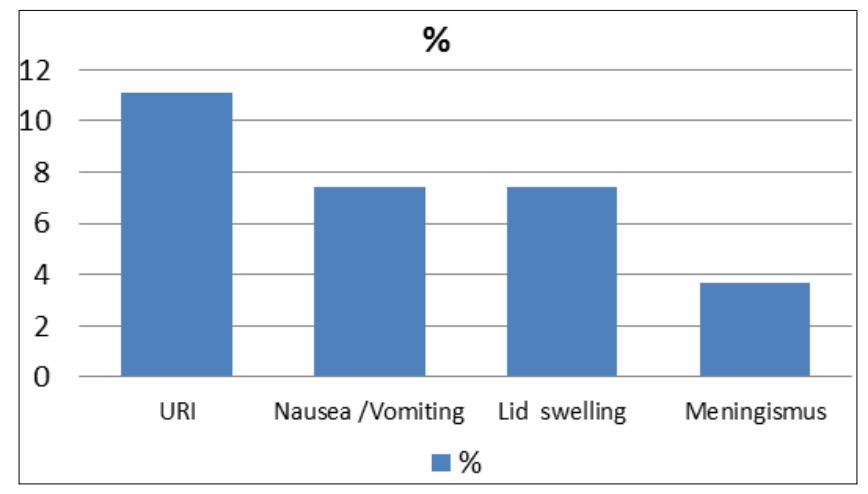

Fig 3: showing complications in peri-operative period

Table 4: Association between variables and complications

\begin{tabular}{|c|c|}
\hline Source & P-Value \\
\hline Age & $0.3174(\mathrm{NS})$ \\
\hline Gender & $0.99(\mathrm{NS})$ \\
\hline Weight & $0.8084(\mathrm{NS})$ \\
\hline Significant past medical illness & $0.99(\mathrm{NS})$ \\
\hline ASA Status & $0.1573(\mathrm{NS})$ \\
\hline H/O URI/LRTI leading Respiratory complications & $0.049(\mathrm{~S})$ \\
\hline
\end{tabular}

\section{Fisher exact chi square test}

There was no association between age, gender, weight, ASA grade and significant past medical illness and complications estimated by statistical analysis with Fischer exact test. Respiratory complications like cough and sneezing were the most common complication in our study. There was more risk of respiratory complications in peri-operative period in patients with history of upper respiratory tract or lower respiratory tract infections $(\mathrm{p}=0.049)$.

\section{Discussion}

In our study, general anaesthesia was well tolerated without any complication during induction, maintenance and emergence. There was no mortality in any child with Cochlear implant. Very few patients had minor complications in our study. Paediatric anaesthesia is sophisticated due to unique anatomical, physiological and pharmacological characteristics ${ }^{1,2}$. Superior intravenous and inhalational inducing agents, better muscle relaxants and advanced anesthesia machine with monitoring devices have made paediatric anaesthesia safer. The youngest child being 1 year 11 months and oldest being 6 years 2 months. All children were healthy belonging to ASA1 and 2. In our study, all complications were very few and occurred in postoperative period. The most common was upper respiratory tract infection in $3(11.11 \%)$ children. Out of these 3 children, 2 children had a history of URI 20 days and 1 month back respectively whereas one child had lower respiratory tract infection 3 weeks back. Because of smaller sized airway and loose mucosa at the cricoid cartilage which is the narrowest part in children less than 7 years, paediatric patients are more prone for laryngospasm, bronchospasm and airway edema. The additional inflammation of airway because of URI/LRTI, may cause airway complications during anaesthesia and post-operatively also. 2 (7.41\%) patients had nausea and vomiting in the post-operative period. These complications were effectively treated without any adverse outcome and were quite similar to percentage in older cochlear implanted patients (major complications $2.3 \%$ to $4.1 \%$ ). Hawksworth C et al. (2015) ${ }^{[3]}$ conducted an audit of anaesthesia safety in paediatric patients of less than
16 years of age. They observed that $40 \%$ children had one or more co-morbidities including $11.9 \%$ were pre-mature babies. Complications were noted in 22 patients, majority were minimal. 2 patients had major complications (rate$0.8 \%$ ). Undeclared upper respiratory tract infection was the most likely greatest preventable cause of complication. They concluded that anaesthesia for cochlear implant in children less than 16 years is safe. Darlong V et al. (2015) [4] conducted a study for perioperative complications of cochlear implant surgery in children of pre lingual and postlingual group. All patients received general anaesthesia. Anaesthesia related complications were laryngospasm at extubation $4.73 \%$, emergence agitation $2.63 \%$ and postoperative nausea and vomiting $1.05 \%$. Major surgical complications were CSF leak without meningitis 3.15\%, device migration or failure $1.05 \%$ and flap infection $1.57 \%$. They concluded that cochlear implant under general anaesthesia in small children was safe and anaesthesia related complications were minimal. In a study conducted by Vlasterakos PV et al. (2010) ${ }^{[4]}$ for diagnostic challenges and safety considerations in cochlear implantation under the age of 12 months, they observed that no major anaesthetic complications in any of the child. The rate of surgical complications was $8.8 \%$ (3.3\% major complications) which was similar to percentage in older implanted patients (major complications $2.3 \%$ to $4.1 \%$ ). They concluded that no increased rate of surgical and anaesthetic complications in infant implantees denoting the safety of surgery and anaesthesia. In a study conducted by Dettman SJ et al. (2007) [6] for the assessment of risk versus benefits in children less than 12 months of cochlear implant, concluded that cochlear implantation can be safely performed within experienced paediatric implantation centre. Chakrabarty A et al. (2004) ${ }^{[7]}$ concluded that no remarkable anaesthetic or surgical complications in cochlear implant patients except perilymph leakage in two cases. Ajallouyean M. et al. (2011) ${ }^{[8]}$ also favour in the safety of anaesthesia technique in cochlear implantees except laryngospasm in 1(5.7\%) patient. Raghunandhan S et al. (2014) ${ }^{[9]}$ concluded that cochlear implant is safe in long term with minimal complications.

\section{Conclusion}

In our study, paediatric patients undergoing Cochlear implantation, general anaesthesia technique was very well tolerated as there was no anaesthetic complication during induction, maintenance and emergence. In post-operative period the most common complication was respiratory which was managed effectively with antibiotics, antihistaminics and Duolin nebulization without any long term sequelae. Based on various findings from our study, we can conclude that with meticulous pre-operative evaluation, systematic planning of anaesthesia technique with strict hemodynamic monitoring and rigorous post-operative management, general anaesthesia is safe in paediatric patients undergoing cochlear implantation.

\section{References}

1. Cote CJ, Ryan JF, Todres ID et al. A practise of anaesthesia for infants and children. Philadelphia, PA: WB Saunders, 1992, 55.

2. Eckenhoff JE. Some anatomic considerations of the infant larynx influencing endotracheal anaesthesia. Anesthesiology. 1951; 12:401-410. 
3. Hawksworth C, Ravery S. An audit of anaesthesia safety in a pediatric cochlear implantation program. Pediatric Anaesthesia. 2015; 26(6):126-30.

4. Darlong V, Khanna P, Baidya DK, Chandralekha, Pandey R, Punj J et al. Perioperative complications of cochlear implant surgery in children. Journal Of Anaesthesia. 2014; 29:126-130.

5. Vlasterakos PV, Caniloros D. Diagnostic challenges and safety considerations in cochlear implantation under the age of 12 months. International Journal Of Pediatric Otorhinologylaryngology. 2010; 74(2):127132.

6. Dettman SJ, Pinder D, Briggs RJS, Dowell RC. Communication development in children who receive the cochlear implant younger than 12 months: risks versus benefits. Ear and hearing. 2007; 28(2):11-18.

7. Chakrabarty A, Tarneja VK, Shrivastava DK. Cochlear implant: Anesthesia Challenges. Medical Journal, Armed Forces India. 2004; 60(4):351-356.

8. Ajallouyean M, Anirsalari S, Yousefi J. A report of surgical complications in a series of 262 consecutive pediatric cochlear implantation in Iran. Iranian Journal of Pediatrics. 2011; 21(4):455-60.

9. Raghunandhan S, Kameshwaran M, Houssain MD. A study of complications and morbidity profile in cochlear implantation: the MERF experience. Indian J Otolaryngol Head Neck Surgery. 2014; 66(1):161-168.

10. Coelho DH, Yeh J, Kim JT, Lalwani AK. Cochlear implantation is associated with minimal anaesthetic risk in elderly. Laryngoscope. 2009; 119(2):355-358.

11. Selby IR, Rigg JD, Faragher B et al. The incidence of minor sequelae following anaesthesia in children. Paediatr Anaesthesiol. 1996; 6:293.

12. Yeh JS, Mooney KL, Gingrich K et al. Anesthetic complications in pediatric patients undergoing cochlear implantation. The Laryngoscope. 2011; 121:2240-2244. 